\title{
CONVERGENCE RESULTS FOR FAMILIES OF UNIVALENT MAPPINGS ON THE UNIT BALL IN $\mathrm{C}^{n}$
}

\author{
Hidetaka Hamada, Mihai Iancu and Gabriela Kohr \\ Kyushu Sangyo University, Faculty of Engineering \\ 3-1 Matsukadai 2-Chome, Higashi-ku Fukuoka 813-8503, Japan; h.hamada@ip.kyusan-u.ac.jp \\ Babeş-Bolyai University, Faculty of Mathematics and Computer Science \\ 1 M. Kogălniceanu Str., 400084 Cluj-Napoca, Romania; miancu@math.ubbcluj.ro \\ Babeş-Bolyai University, Faculty of Mathematics and Computer Science \\ 1 M. Kogălniceanu Str., 400084 Cluj-Napoca, Romania; gkohr@math.ubbcluj.ro
}

\begin{abstract}
Let $\widetilde{S}_{A}^{t}\left(\mathbf{B}^{n}\right)$ be the family of normalized univalent mappings on the Euclidean unit ball $\mathbf{B}^{n}$ in $\mathbf{C}^{n}$, which have generalized parametric representation with respect to time-dependent operators $A \in \widetilde{\mathcal{A}}$, where $\widetilde{\mathcal{A}}$ is a family of measurable mappings from $[0, \infty)$ into $L\left(\mathbf{C}^{n}\right)$ with some particular properties. Also, let $\widetilde{\mathcal{R}}_{T}\left(\operatorname{id}_{\mathbf{B}^{n}},\left(\mathcal{N}_{A(t)}\right)_{t \in\left[T_{0}, T\right]}\right)$ be the time-T-reachable family of the control system $\mathcal{C}\left(\left[T_{0}, T\right],\left(\mathcal{N}_{A(t)}\right)_{t \in\left[T_{0}, T\right]}\right)$, where $A \in \widetilde{\mathcal{A}}$ and $T_{0} \geq 0$. In this paper we obtain certain convergence results for the families $\widetilde{S}_{A}^{t}\left(\mathbf{B}^{n}\right)$ and $\widetilde{\mathcal{R}}_{T}\left(\operatorname{id}_{\mathbf{B}^{n}},\left(\mathcal{N}_{A(t)}\right)_{t \in\left[T_{0}, T\right]}\right)$ with respect to the Hausdorff metric $\rho$ on $H\left(\mathbf{B}^{n}\right)$. These results may be seen as dominated convergence type theorems for time-dependent operators $A \in \widetilde{\mathcal{A}}$. In particular, we obtain related convergence results for the family $S_{\mathbf{A}}^{0}\left(\mathbf{B}^{n}\right)$ (resp. for the family $\widehat{S}_{\mathbf{A}}\left(\mathbf{B}^{n}\right)$ ) of mappings with A-parametric representation on $\mathbf{B}^{n}$ (resp. of spirallike mappings on $\mathbf{B}^{n}$ with respect to $\mathbf{A}$ ), in the case that $\mathbf{A} \in L\left(\mathbf{C}^{n}\right)$ is a linear operator with $k_{+}(\mathbf{A})<2 m(\mathbf{A})$, where $k_{+}(\mathbf{A})$ is the Lyapunov index of $\mathbf{A}$ and $m(\mathbf{A})=$ $\min _{\|z\|=1} \Re\langle\mathbf{A}(z), z\rangle$. We also obtain a convergence result for the Carathéodory family $\mathcal{N}_{\mathbf{A}}$, where $m(\mathbf{A})>0$. Finally, we obtain some sufficient conditions related to $A \in \widetilde{\mathcal{A}}$, which yield the equality $\widetilde{S}_{A}^{t}\left(\mathbf{B}^{n}\right)=S^{0}\left(\mathbf{B}^{n}\right)$, for all $t \geq 0$, where $S^{0}\left(\mathbf{B}^{n}\right)$ is the family of normalized univalent mappings with usual parametric representation on $\mathbf{B}^{n}$. Certain consequences are also provided.
\end{abstract}

\section{Introduction}

Since the early works devoted to Loewner chains and the Loewner differential equation in higher dimensions due to Pfaltzgraff [27] and Poreda [28, 29], many results in this field have been obtained (see $[1,5,6,9,11,13,14,15,20,21,35]$ ). We also mention the main contributions of Bracci [5] related to the existence of bounded support points for the family $S^{0}\left(\mathbf{B}^{n}\right), n \geq 2$, and of Roth [31] concerning the $n$ dimensional version of the well known Pontryagin maximum principle. Other recent contributions in the Loewner theory in $\mathbf{C}^{n}$ may be found in $[2,3,4,7,16,17,23,24$, $25,32]$.

doi:10.5186/aasfm.2016.4136

2010 Mathematics Subject Classification: Primary 32H02; Secondary 30C45.

Key words: Carathéodory family, Hausdorff metric, Loewner chain, parametric representation, reachable family, transition mapping.

H. Hamada was partially supported by JSPS KAKENHI Grant Number 25400151.

M. Iancu was supported by SOP HRD 2007-2013, co-financed by the European Social Fund, under the project POSDRU/159/1.5/S/132400.

G. Kohr was supported by a grant of the Romanian National Authority for Scientific Research, CNCS-UEFISCDI, project number PN-II-ID-PCE-2011-3-0899. 
Let $\widetilde{\mathcal{A}}$ be the family of all measurable mappings $A:[0, \infty) \rightarrow L\left(\mathbf{C}^{n}\right)$, which satisfy the following conditions:

(i) $m(A(\tau)) \geq 0$, for a.e. $\tau \geq 0$;

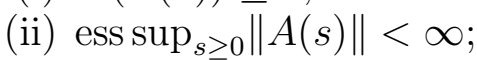

(iii) $\sup _{s \geq 0} \int_{s}^{\infty}\left\|V(s, t)^{-1}\right\| e^{-2 \int_{s}^{t} m(A(\tau)) d \tau} d t<\infty$, where $V(s, t)$ is the unique solution on $[s, \infty)$ of the initial value problem $(2.1)$.

The authors in [22] have investigated various extremal properties of compact families $\widetilde{S}_{A}^{t}\left(\mathbf{B}^{n}\right)(t \geq 0)$ consisting of normalized biholomorphic mappings on the Euclidean unit ball $\mathbf{B}^{n}$ in $\mathbf{C}^{n}$ which have generalized parametric representation with respect to time-dependent linear operators $A \in \widetilde{\mathcal{A}}$. We have considered examples and applications which yield that the study of the family $\widetilde{S}_{A}^{t}\left(\mathbf{B}^{n}\right)$ for time-dependent operators $A \in \widetilde{\mathcal{A}}$ is basically different from that in the case of constant time-dependent linear operators (see [22]). In the case that $A(t)=\mathbf{A}$, for all $t \geq 0$, where $\mathbf{A} \in L\left(\mathbf{C}^{n}\right)$ with $k_{+}(\mathbf{A})<2 m(\mathbf{A})$, then $\widetilde{S}_{A}^{t}\left(\mathbf{B}^{n}\right)=S_{\mathbf{A}}^{0}\left(\mathbf{B}^{n}\right)$, for all $t \geq 0$, where $S_{\mathbf{A}}^{0}\left(\mathbf{B}^{n}\right)$ is the family of mappings with $\mathbf{A}$-parametric representation (see [13]). Note that $k_{+}(\mathbf{A})$ is the Lyapunov index of $\mathbf{A}$ and $m(\mathbf{A})=\min _{\|z\|=1} \Re\langle\mathbf{A}(z), z\rangle$. If $n=1$ and $a \in \widetilde{A}$, then $\widetilde{S}_{a}^{t}(\mathbf{U})=S$, for all $t \geq 0$ (see [22]), where $S$ is the family of normalized univalent functions on the unit disc $\mathbf{U}$.

In this paper we consider a certain dependence of the family $\widetilde{S}_{A}^{T}\left(\mathbf{B}^{n}\right)$ on $A \in \widetilde{\mathcal{A}}$, where $T \geq 0$. The main results of this paper can be summarized as follows. The notations in the following results will be explained in the next sections.

Theorem 1.1. Let $T \geq 0$ and $A \in \widetilde{\mathcal{A}}$ be such that $\operatorname{ess} \inf _{t \geq T} m(A(t))>0$. Also, let $M>0, \alpha \in L^{1}([T, \infty), \mathbf{R})$ and $\left(A_{k}\right)_{k \in \mathbf{N}}$ be a sequence in $\widetilde{\mathcal{A}}$ such that $\left\|A_{k}(t)\right\| \leq M$ and $\left\|V_{k}(T, t)^{-1}\right\| e^{-2 \int_{T}^{t} m\left(A_{k}(\tau)\right) d \tau} \leq \alpha(t)$, for a.e. $t \geq T$ and for all $k \in \mathbf{N}$, where $V_{k}(T, \cdot)$ is the unique solution on $[T, \infty)$ of the initial value problem (2.1) related to $A_{k}$. If $\lim _{k \rightarrow \infty} A_{k}(t)=A(t)$ for a.e. $t \geq T$, then $\lim _{k \rightarrow \infty} \rho\left(\widetilde{S}_{A_{k}}^{T}\left(\mathbf{B}^{n}\right), \widetilde{S}_{A}^{T}\left(\mathbf{B}^{n}\right)\right)=0$.

Theorem 1.2. Let $\mathbf{A} \in L\left(\mathbf{C}^{n}\right)$ be such that $k_{+}(\mathbf{A})<2 m(\mathbf{A})$, and let $\left(\mathbf{A}_{l}\right)_{l \in \mathbf{N}}$ be a sequence in $L\left(\mathbf{C}^{n}\right)$ such that $\mathbf{A}_{l} \rightarrow \mathbf{A}$, as $l \rightarrow \infty$. Then there is $l_{0} \in \mathbf{N}$ such that $S_{\mathbf{A}_{l}}^{0}\left(\mathbf{B}^{n}\right)$ is compact for $l \geq l_{0}$, and $\rho\left(S_{\mathbf{A}_{l}}^{0}\left(\mathbf{B}^{n}\right), S_{\mathbf{A}}^{0}\left(\mathbf{B}^{n}\right)\right) \rightarrow 0$, as $l \rightarrow \infty$.

In view of the definition of the family $\widetilde{\mathcal{A}}$, it follows that Theorem 1.1 may be seen as a dominated convergence type theorem. In particular, we obtain a related convergence result for the compact family $\widehat{S}_{\mathbf{A}}\left(\mathbf{B}^{n}\right)$ consisting of spirallike mappings on $\mathbf{B}^{n}$ with respect to $\mathbf{A}$, in the case that $\mathbf{A} \in L\left(\mathbf{C}^{n}\right)$ is a constant time-dependent linear operator with $k_{+}(\mathbf{A})<2 m(\mathbf{A})$. We also obtain a convergence result related to the Carathéodory family $\mathcal{N}_{\mathbf{A}}$, where $m(\mathbf{A})>0$.

The authors in [22] obtained extremal properties for the family $\widetilde{S}_{A}^{t}\left(\mathbf{B}^{n}\right)$ consisting of normalized univalent mappings on $\mathbf{B}^{n}$ which have generalized parametric representation with respect to time-dependent operators $A \in \widetilde{\mathcal{A}}$, and deduced certain applications by considering examples of time-dependent normalizations that are step functions. In this paper we shall apply Theorem 1.1 to obtain other results which involve time-dependent operators that are step functions. For example, in the last section we shall obtain some sufficient conditions for a time-dependent operator $A \in \widetilde{\mathcal{A}}$ such that $\widetilde{S}_{A}^{t}\left(\mathbf{B}^{n}\right)=S^{0}\left(\mathbf{B}^{n}\right)$, for all $t \geq 0$, where $S^{0}\left(\mathbf{B}^{n}\right)$ is the family of normalized univalent mappings with usual parametric representation on $\mathbf{B}^{n}$. 


\section{Preliminaries}

Let $\mathbf{C}^{n}$ be the space of $n$ complex variables $z=\left(z_{1}, \ldots, z_{n}\right)$ with the Euclidean inner product $\langle z, w\rangle=\sum_{j=1}^{n} z_{j} \bar{w}_{j}$ and the Euclidean norm $\|z\|=\langle z, z\rangle^{1 / 2}$. The open ball $\left\{z \in \mathbf{C}^{n}:\|z\|<r\right\}$ is denoted by $\mathbf{B}_{r}^{n}$ and the unit ball $\mathbf{B}_{1}^{n}$ is denoted by $\mathbf{B}^{n}$. The closed ball $\left\{z \in \mathbf{C}^{n}:\|z\| \leq r\right\}$ is denoted by $\overline{\mathbf{B}_{r}^{n}}$. In the case $n=1$, the unit $\operatorname{disc} \mathbf{B}^{1}$ is denoted by $\mathbf{U}$.

Let $L\left(\mathbf{C}^{n}\right)$ denote the space of linear operators from $\mathbf{C}^{n}$ into $\mathbf{C}^{n}$ with the standard operator norm. Also, let $I_{n}$ be the identity operator in $L\left(\mathbf{C}^{n}\right)$. If $A \in L\left(\mathbf{C}^{n}\right)$, we denote by $A^{*}$ the adjoint of the operator $A$. Let $H\left(\mathbf{B}^{n}\right)$ be the family of holomorphic mappings from $\mathbf{B}^{n}$ into $\mathbf{C}^{n}$ with the compact-open topology. If $f \in H\left(\mathbf{B}^{n}\right)$, we say that $f$ is normalized if $f(0)=0$ and $D f(0)=I_{n}$. Let $S\left(\mathbf{B}^{n}\right)$ be the family of normalized biholomorphic mappings on $\mathbf{B}^{n}$. If $n=1$, then the family $S(\mathbf{U})$ is denoted by $S$.

Next, we use the following notations for an operator $A \in L\left(\mathbf{C}^{n}\right)$ (see e.g. [10, 13]):

$$
\begin{aligned}
m(A) & =\min \{\Re\langle A(z), z\rangle:\|z\|=1\}, \\
k(A) & =\max \{\Re\langle A(z), z\rangle:\|z\|=1\}, \\
|V(A)| & =\max \{|\langle A(z), z\rangle|:\|z\|=1\}, \\
k_{+}(A) & =\max \{\Re \lambda: \lambda \in \sigma(A)\},
\end{aligned}
$$

where $\sigma(A)$ is the spectrum of $A$. Note that $|V(A)|$ is the numerical radius of the operator $A$ and $k_{+}(A)$ is the upper exponential index (Lyapunov index) of $A$. Then $m(A) \leq k_{+}(A) \leq|V(A)| \leq\|A\|$ (see e.g. [16]) and it is known that $\|A\| \leq 2|V(A)|$ and $k_{+}(A)=\lim _{t \rightarrow \infty} \frac{\log \left\|e^{t A}\right\|}{t}$ (see e.g. [10]).

The following families of holomorphic mappings on the unit ball $\mathbf{B}^{n}$ play the role of the Carathéodory family in $\mathbf{C}^{n}$ (see [33]):

$$
\begin{aligned}
& \mathcal{N}=\left\{h \in H\left(\mathbf{B}^{n}\right): h(0)=0, \Re\langle h(z), z\rangle \geq 0, z \in \mathbf{B}^{n}\right\}, \\
& \widetilde{\mathcal{N}}=\left\{h \in \mathcal{N}: \Re\langle h(z), z\rangle>0, z \in \mathbf{B}^{n} \backslash\{0\}\right\} .
\end{aligned}
$$

If $A \in L\left(\mathbf{C}^{n}\right)$ with $m(A) \geq 0$, let (see e.g. [13])

$$
\mathcal{N}_{A}=\{h \in \mathcal{N}: \operatorname{Dh}(0)=A\} .
$$

Also, let $\mathcal{M}=\mathcal{N}_{I_{n}}$. In view of the minimum principle for harmonic mappings, it is easily seen that (see [33])

$$
\mathcal{M}=\left\{h \in \tilde{\mathcal{N}}: D h(0)=I_{n}\right\} .
$$

The following growth result was obtained by Graham, Hamada, and Kohr [11] for the family $\mathcal{M}$ (see [13, Lemma 1.2] in the case of mappings $h \in \widetilde{\mathcal{N}}$; see also [34, Proposition 1.2.3] for the family $\mathcal{N}$ ).

Lemma 2.1. If $h \in \mathcal{N}$, then

$$
\|h(z)\| \leq \frac{4\|z\|}{(1-\|z\|)^{2}}|V(D h(0))|, \quad z \in \mathbf{B}^{n} .
$$

Next, let $A:[0, \infty) \rightarrow L\left(\mathbf{C}^{n}\right)$ be a measurable mapping which is locally integrable on $[0, \infty)$. For every $s \geq 0$, let $V(s, \cdot):[s, \infty) \rightarrow L\left(\mathbf{C}^{n}\right)$ be the unique locally absolutely continuous solution of the initial value problem (cf. [34])

$$
\frac{\partial V}{\partial t}(s, t)=-A(t) V(s, t), \text { a.e. } t \in[s, \infty), V(s, s)=I_{n}
$$


Also, let $V(t)=V(0, t)$, for all $t \geq 0$. Then $V(s, t)=V(t) V(s)^{-1}$ for $0 \leq s \leq t<\infty$ (see [8]; cf. [34]).

Remark 2.2. Let $s \geq 0$. If $A(t)$ and $\int_{s}^{t} A(\tau) d \tau$ commute for $t \geq s$, then

$$
V(s, t)=e^{-\int_{s}^{t} A(\tau) d \tau}, \quad \forall t \in[s, \infty),
$$

by [8, Exercise VII.2.22].

The following estimates related to a measurable and locally integrable mapping $A:[0, \infty) \rightarrow L\left(\mathbf{C}^{n}\right)$ will be useful in the forthcoming sections (see [34, Proposition 1.2.1, Remark 1.2.2]; cf. [14, Remark $1.6(\mathrm{v})]$ ).

Lemma 2.3. Let $A:[0, \infty) \rightarrow L\left(\mathbf{C}^{n}\right)$ be a measurable mapping that is locally integrable, and let $V(s, t)$ be the unique solution on $[s, \infty)$ of the initial value problem (2.1) related to $A$. Then

$$
e^{\int_{s}^{t} m(A(\tau)) d \tau} \leq\left\|V(s, t)^{-1}\right\| \leq e^{\int_{s}^{t} k(A(\tau)) d \tau}
$$

and

for all $t \geq s \geq 0$.

$$
e^{-\int_{s}^{t} k(A(\tau)) d \tau} \leq\|V(s, t)\| \leq e^{-\int_{s}^{t} m(A(\tau)) d \tau},
$$

Next, we recall the notion of generalized parametric representation with respect to a time-dependent linear operator (see [22]; cf. [14, Definition 1.5], [34, Proposition 1.5.1]).

Definition 2.4. Let $A:[0, \infty) \rightarrow L\left(\mathbf{C}^{n}\right)$ be a measurable mapping, which is locally integrable, such that $m(A(t)) \geq 0$ for a.e. $t \geq 0$, and let $T \geq 0$. Also, let $V(s, t)$ be the unique solution on $[s, \infty)$ of the initial value problem (2.1) related to $A$. We say that a mapping $f: \mathbf{B}^{n} \rightarrow \mathbf{C}^{n}$ has generalized parametric representation with respect to $A$ on $[T, \infty)$ if there exists a mapping $h=h(z, t): \mathbf{B}^{n} \times[0, \infty) \rightarrow \mathbf{C}^{n}$ which satisfies the following conditions:

(i) $h(z, \cdot)$ is measurable on $[0, \infty)$, for all $z \in \mathbf{B}^{n}$;

(ii) $h(\cdot, t) \in \mathcal{N}$, for all $t \geq 0$;

(iii) $D h(0, t)=A(t)$, for all $t \geq 0$;

and such that

$$
f(z)=\lim _{t \rightarrow \infty} V(T, t)^{-1} v(z, T, t)
$$

locally uniformly on $\mathbf{B}^{n}$, where $v(z, T, \cdot):[T, \infty) \rightarrow \mathbf{C}^{n}$ is the unique locally absolutely continuous solution of the initial value problem

$$
\frac{\partial v}{\partial t}(z, T, t)=-h(v(z, T, t), t) \text {, a.e. } t \in[T, \infty), v(z, T, T)=z,
$$

for all $z \in \mathbf{B}^{n}$. Let $\widetilde{S}_{A}^{T}\left(\mathbf{B}^{n}\right)$ be the family of mappings with generalized parametric representation with respect to $A$ on $[T, \infty)$.

Obviously, $\widetilde{S}_{A}^{T}\left(\mathbf{B}^{n}\right) \neq \emptyset$, since $\operatorname{id}_{\mathbf{B}^{n}} \in \widetilde{S}_{A}^{T}\left(\mathbf{B}^{n}\right)$, for $T \geq 0$ and every measurable and locally integrable mapping $A:[0, \infty) \rightarrow L\left(\mathbf{C}^{n}\right)$ such that $m(A(t)) \geq 0$, for a.e. $t \geq 0$.

Definition 2.5. Let $A:[0, \infty) \rightarrow L\left(\mathbf{C}^{n}\right)$ be a measurable mapping, which is locally integrable on $[0, \infty)$, such that $m(A(t)) \geq 0$, for a.e. $t \geq 0$. A mapping $h: \mathbf{B}^{n} \times[0, \infty) \rightarrow \mathbf{C}^{n}$ which satisfies the conditions (i)-(iii) of Definition 2.4 will be called a Herglotz vector field (or a generating vector field) with respect to $A$ (cf. [6] and $[9])$. 
Remark 2.6. Let $\mathbf{A} \in L\left(\mathbf{C}^{n}\right)$ be such that $m(\mathbf{A})>0$ and let $A:[0, \infty) \rightarrow L\left(\mathbf{C}^{n}\right)$ be such that $A(t)=\mathbf{A}$, for all $t \geq 0$. In this case, the family $\widetilde{S}_{A}^{t}\left(\mathbf{B}^{n}\right)$ reduces to the family $S_{\mathbf{A}}^{0}\left(\mathbf{B}^{n}\right)$ of mappings with $\mathbf{A}$-parametric representation on $\mathbf{B}^{n}$, for all $t \geq 0$ (see [13]). If $\mathbf{A}=I_{n}$, then $S_{\mathbf{A}}^{0}\left(\mathbf{B}^{n}\right)=S^{0}\left(\mathbf{B}^{n}\right)$, where $S^{0}\left(\mathbf{B}^{n}\right)$ is the family of mappings with the usual parametric representation on $\mathbf{B}^{n}$ (see [11] and [13]).

Various properties of mappings with generalized parametric representation may be found in [12], [14], and [22].

Definition 2.7. (see [33]) Let $A \in L\left(\mathbf{C}^{n}\right)$ be such that $m(A)>0$. A mapping $f \in S\left(\mathbf{B}^{n}\right)$ is said to be spirallike with respect to $A$ (denoted by $f \in \widehat{S}_{A}\left(\mathbf{B}^{n}\right)$ ) if $e^{-t A} f\left(\mathbf{B}^{n}\right) \subseteq f\left(\mathbf{B}^{n}\right)$, for all $t \geq 0$.

Next we recall the notion of a univalent subordination chain whose normalization is given by a time-dependent linear operator in $\mathbf{C}^{n}$ (see [14]; cf. [18, Chpater 8]).

Definition 2.8. A mapping $f: \mathbf{B}^{n} \times[0, \infty) \rightarrow \mathbf{C}^{n}$ is called a univalent subordination chain if $f(\cdot, t)$ is univalent on $\mathbf{B}^{n}, f(0, t)=0$ for $t \geq 0$, and $f\left(\mathbf{B}^{n}, s\right) \subseteq f\left(\mathbf{B}^{n}, t\right)$ for $0 \leq s \leq t$. If, in addition, $D f(0, t)=V(t)^{-1}$ for $t \geq 0$, and $\{V(t) f(\cdot, t)\}_{t \geq 0}$ is a normal family on $\mathbf{B}^{n}$, then we say that $f$ is a normal Loewner chain with respect to $A$, where $A:[0, \infty) \rightarrow L\left(\mathbf{C}^{n}\right)$ is a measurable and locally integrable mapping and $V(t)=V(0, t)$ is the unique solution on $[0, \infty)$ of the initial value problem $(2.1)$ related to $A$.

Note that if $f=f(z, t)$ is a univalent subordination chain, then there exists a unique univalent Schwarz mapping $v=v(z, s, t)$, called the transition mapping associated with $f$, such that

$$
f(z, s)=f(v(z, s, t), t), \quad z \in \mathbf{B}^{n}, 0 \leq s \leq t<\infty .
$$

The family $\left(v_{s, t}\right)$ is also called the evolution family associated with $f(z, t)$, where $v_{s, t}(z)=v(z, s, t)$ (cf. [6]).

Remark 2.9. It is easily seen that if $\mathbf{A} \in L\left(\mathbf{C}^{n}\right)$ and $f \in H\left(\mathbf{B}^{n}\right)$ is a normalized mapping, then $f \in \widehat{S}_{\mathbf{A}}\left(\mathbf{B}^{n}\right)$ if and only if $f(z, t)=e^{t \mathbf{A}} f(z)$ is a normal Loewner chain with respect to $\mathbf{A}$ (see [13]).

In this paper we are concerned with normal Loewner chains whose normalizations depend on operators $A \in \widetilde{\mathcal{A}}$, where $\widetilde{\mathcal{A}}$ is the family of operators $A:[0, \infty) \rightarrow L\left(\mathbf{C}^{n}\right)$ given in Definition 2.10 below (see [22]):

Definition 2.10. Let $\widetilde{\mathcal{A}}$ be the family of all measurable mappings $A:[0, \infty) \rightarrow$ $L\left(\mathbf{C}^{n}\right)$, which satisfy the following conditions:

(i) $m(A(\tau)) \geq 0$, for a.e. $\tau \geq 0$;

(ii) $\operatorname{ess} \sup _{s \geq 0}\|A(s)\|<\infty$;

(iii) $\sup _{s \geq 0} \int_{s}^{\infty}\left\|V(s, t)^{-1}\right\| e^{-2 \int_{s}^{t} m(A(\tau)) d \tau} d t<\infty$,

where $V(s, t)$ is the unique solution on $[s, \infty)$ of the initial value problem $(2.1)$ related to $A$.

Remark 2.11. Let $T>0, \mathbf{A} \in L\left(\mathbf{C}^{n}\right)$ and let $A:[0, \infty) \rightarrow L\left(\mathbf{C}^{n}\right)$ be such that $m(A(t)) \geq 0$, for a.e. $t \in[0, T]$, $\operatorname{ess} \sup _{t \in[0, T]}\|A(t)\|<\infty$ and $A(t)=\mathbf{A}$, for a.e. $t>T$. Then $A \in \widetilde{\mathcal{A}}$ if and only if $k_{+}(\mathbf{A})<2 m(\mathbf{A})$, by Lemma 2.3, [9, Remark 2.8] and $\left[14\right.$, Remark 2.2]. In particular, $I_{n} \in \tilde{\mathcal{A}}$. 
Remark 2.12. (i) The authors in [22] proved that if $T \geq 0, A \in \widetilde{\mathcal{A}}$, and $g \in$ $H\left(\mathbf{B}^{n}\right)$ is a normalized mapping, then $g \in \widetilde{S}_{A}^{T}\left(\mathbf{B}^{n}\right)$ if and only if there exists a normal Loewner chain $f=f(z, t)$ with respect to $A$ such that $g=V(T) f(\cdot, T)$, where $V(t)=V(0, t)$ is the unique locally absolutely continuous solution on $[0, \infty)$ of the initial value problem (2.1) related to $A$. In particular, if $\mathbf{A} \in L\left(\mathbf{C}^{n}\right)$ is a constant time-dependent operator such that $k_{+}(\mathbf{A})<2 m(\mathbf{A})$, then $f \in S_{\mathbf{A}}^{0}\left(\mathbf{B}^{n}\right)$ if and only if there is a normal Loewner chain $f(z, t)$ with respect to $\mathbf{A}$ such that $f=f(\cdot, 0)$ (see [13]).

(ii) If $\mathbf{A} \in L\left(\mathbf{C}^{n}\right)$ with $k_{+}(\mathbf{A})<2 m(\mathbf{A})$, then $\widehat{S}_{\mathbf{A}}\left(\mathbf{B}^{n}\right)$ is a compact family in $H\left(\mathbf{B}^{n}\right)$ (see [35]; cf. [13]) and $\widehat{S}_{\mathbf{A}}\left(\mathbf{B}^{n}\right) \subset S_{\mathbf{A}}^{0}\left(\mathbf{B}^{n}\right)$ (see [13] and [35]).

The results contained in Proposition 2.13 and Lemma 2.14 were obtained in [22].

Proposition 2.13. Let $a:[0, \infty) \rightarrow \mathbf{R}$ be a measurable function such that

$$
\operatorname{essinf}_{t \geq 0} a(t)>0 \text { and }{\operatorname{ess} \sup _{t \geq 0} a(t)<\infty .}
$$

Also, let $\mathbf{A} \in L\left(\mathbf{C}^{n}\right)$ be such that $k_{+}(\mathbf{A})<2 m(\mathbf{A})$ and let $A:[0, \infty) \rightarrow L\left(\mathbf{C}^{n}\right)$ be given by $A(t)=a(t) \mathbf{A}$ for a.e. $t \geq 0$. Then $A \in \widetilde{\mathcal{A}}$ and $\widetilde{S}_{A}^{T}\left(\mathbf{B}^{n}\right)=S_{\mathbf{A}}^{0}\left(\mathbf{B}^{n}\right)$ for $T \geq 0$.

Lemma 2.14. Let $T \geq 0$ and $A \in \widetilde{\mathcal{A}}$. Also, let $f$ be a normal Loewner chain with respect to $A$, and let $v$ be the transition mapping associated with $f$. If $h \in \widetilde{S}_{A}^{T}\left(\mathbf{B}^{n}\right)$, then $V(t, T)^{-1} h(v(\cdot, t, T)) \in \widetilde{S}_{A}^{t}\left(\mathbf{B}^{n}\right)$, for all $t \in[0, T]$. In particular, $V(t, T)^{-1} v(\cdot, t, T) \in \widetilde{S}_{A}^{t}\left(\mathbf{B}^{n}\right)$, for all $t \in[0, T]$, where $V(t)=V(0, t)$ and $V(s, t)$ is the unique solution on $[s, \infty)$ of the initial value problem (2.1) related to $A$.

Next, we mention the following growth result for the transition mappings of normal Loewner chains with respect to $A \in \widetilde{\mathcal{A}}$ (see the proof of [22, Proposition 3.10]; cf. [34, Proposition 1.5.2]).

Lemma 2.15. Let $A \in \widetilde{\mathcal{A}}$ and let $f$ be a normal Loewner chain with respect to $A$. Also, let $v$ be the transition mapping associated to $f$. Then for every $r \in(0,1)$, there exists some $C_{r}>0$ such that

$$
\begin{aligned}
& \left\|V\left(s, t_{2}\right)^{-1} v\left(z, s, t_{2}\right)-V\left(s, t_{1}\right)^{-1} v\left(z, s, t_{1}\right)\right\| \\
& \leq C_{r} \int_{t_{1}}^{t_{2}}\|A(t)\|\left\|V(s, t)^{-1}\right\| e^{-2 \int_{s}^{t} m(A(\tau)) d \tau} d t,
\end{aligned}
$$

for all $z \in \overline{\mathbf{B}_{r}^{n}}, s \geq 0$ and $s \leq t_{1}<t_{2} \leq \infty$, where $V(s)=V(0, s)$ and $V(s, t)$ is the unique solution on $[s, \infty)$ of the initial value problem (2.1) related to $A$.

We recall the following definitions that have been recently introduced in [22] (cf. [15, 16, 24, 25]; cf. [30], in the case $n=1$ ).

Definition 2.16. Let $I$ be an interval and $A \in \widetilde{\mathcal{A}}$. A mapping $h: \mathbf{B}^{n} \times I \rightarrow \mathbf{C}^{n}$ is called a Carathéodory mapping on $I$ with respect to $A$ if the following conditions hold:

(i) $h(\cdot, t) \in \mathcal{N}_{A(t)}$, for all $t \in I$;

(ii) $h(z, \cdot)$ is measurable on $I$, for all $z \in \mathbf{B}^{n}$.

Let $\mathcal{C}\left(I,\left(\mathcal{N}_{A(t)}\right)_{t \in I}\right)$ denote the family of Carathéodory mappings on $I$ with respect to $A$. We say that the Carathéodory mappings on $I$ with respect to $A$ represent the controls of the control system $\mathcal{C}\left(I,\left(\mathcal{N}_{A(t)}\right)_{t \in I}\right)$, and $\left(\mathcal{N}_{A(t)}\right)_{t \in I}$ represents the input family. 
Definition 2.17. Let $I$ be either the interval $\left[T_{0}, T_{1}\right]$, where $T_{1}>T_{0} \geq 0$, or the interval $\left[T_{0}, \infty\right)$, where $T_{0} \geq 0$, and $A \in \widetilde{\mathcal{A}}$. For every $h \in \mathcal{C}\left(I,\left(\mathcal{N}_{A(t)}\right)_{t \in I}\right)$ we denote by $v\left(z, T_{0}, \cdot ; h\right)$ the unique locally absolutely continuous solution on $I$ of the initial value problem

$$
\left\{\begin{array}{l}
\frac{\partial v}{\partial t}\left(z, T_{0}, t ; h\right)=-h\left(v\left(z, T_{0}, t ; h\right), t\right), \quad \text { for a.e. } t \in I \\
v\left(z, T_{0}, T_{0} ; h\right)=z
\end{array}\right.
$$

for all $z \in \mathbf{B}^{n}$.

Note that $v\left(\cdot, T_{0}, t ; h\right)$ is a univalent Schwarz mapping with $D v\left(0, T_{0}, t ; h\right)=$ $V\left(T_{0}, t\right)$, for all $t \in I$ (cf. [34] and [22]), where $V\left(T_{0}, \cdot\right)$ is the unique solution on $\left[T_{0}, \infty\right)$ of the initial value problem $(2.1)$ related to $A$.

Now, we consider the notion of the reachable family with respect to time-dependent linear operators (see [22]).

Definition 2.18. Let $T_{0} \geq 0$ and $A \in \widetilde{\mathcal{A}}$. For every $T>T_{0}$ we denote the normalized time-T-reachable family of the control system $\mathcal{C}\left(\left[T_{0}, T\right],\left(\mathcal{N}_{A(t)}\right)_{t \in\left[T_{0}, T\right]}\right)$ by $\widetilde{\mathcal{R}}_{T}\left(\operatorname{id}_{\mathbf{B}^{n}},\left(\mathcal{N}_{A(t)}\right)_{t \in\left[T_{0}, T\right]}\right)=\left\{V\left(T_{0}, T\right)^{-1} v\left(\cdot, T_{0}, T ; h\right): h \in \mathcal{C}\left(\left[T_{0}, T\right],\left(\mathcal{N}_{A(t)}\right)_{t \in\left[T_{0}, T\right]}\right)\right\}$.

We also denote the normalized infinite-time-reachable family of the control system $\mathcal{C}\left(\left[T_{0}, \infty\right),\left(\mathcal{N}_{A(t)}\right)_{t \geq T_{0}}\right)$ by

$$
\widetilde{\mathcal{R}}_{\infty}\left(\operatorname{id}_{\mathbf{B}^{n}},\left(\mathcal{N}_{A(t)}\right)_{t \geq T_{0}}\right)=\left\{\lim _{t \rightarrow \infty} V\left(T_{0}, t\right)^{-1} v\left(\cdot, T_{0}, t ; h\right): h \in \mathcal{C}\left(\left[T_{0}, \infty\right),\left(\mathcal{N}_{A(t)}\right)_{t \geq T_{0}}\right)\right\} .
$$

Remark 2.19. In view of Definition 2.4 and Lemma 2.14 (ii), we have that $\widetilde{\mathcal{R}}_{\infty}\left(\operatorname{id}_{\mathbf{B}^{n}},\left(\mathcal{N}_{A(t)}\right)_{t \geq T_{0}}\right)=\widetilde{S}_{A}^{T_{0}}\left(\mathbf{B}^{n}\right)$ and $\widetilde{\mathcal{R}}_{T}\left(\operatorname{id}_{\mathbf{B}^{n}},\left(\mathcal{N}_{A(t)}\right)_{t \in\left[T_{0}, T\right]}\right) \subseteq \widetilde{S}_{A}^{T_{0}}\left(\mathbf{B}^{n}\right)$, for all $T \in\left(T_{0}, \infty\right)($ see $[22])$.

Using arguments similar to those in the proofs of [16, Lemmas 4.12 and 4.13] (see [30, Theorem I.29, Lemma I.37] and [25, Proposition 2.3, Lemmas 3.1 and 3.2]), we obtain the following lemmas. We omit the proofs of Lemmas 2.20 and 2.21.

Lemma 2.20. Let $I$ be the interval $\left[T_{0}, T\right]$, where $T>T_{0} \geq 0$, and let $A \in \widetilde{\mathcal{A}}$. Also, let $\left(h_{k}\right)_{k \in \mathbf{N}}$ be a sequence in $\mathcal{C}\left(I,\left(\mathcal{N}_{A(t)}\right)_{t \in I}\right)$. Then there exist a subsequence $\left(h_{k_{m}}\right)_{m \in \mathbf{N}}$ of $\left(h_{k}\right)_{k \in \mathbf{N}}$ and $h \in \mathcal{C}\left(I,\left(\mathcal{N}_{A(t)}\right)_{t \in I}\right)$ such that

$$
\int_{T_{0}}^{t} h_{k_{m}}\left(v\left(\cdot, T_{0}, \tau ; h\right), \tau\right) d \tau \rightarrow \int_{T_{0}}^{t} h\left(v\left(\cdot, T_{0}, \tau ; h\right), \tau\right) d \tau, \quad \text { as } m \rightarrow \infty,
$$

locally uniformly on $\mathbf{B}^{n}$, for all $t \in I$.

Lemma 2.21. Let $I$ be the interval $\left[T_{0}, T\right]$, where $T>T_{0} \geq 0$, let $A \in \widetilde{\mathcal{A}}$, $M>0$, and let $\left(A_{k}\right)_{k \in \mathbf{N}}$ be a sequence in $\widetilde{\mathcal{A}}$ such that $\left\|A_{k}(t)\right\| \leq M$, for a.e. $t \in I$ and for all $k \in \mathbf{N}$. Let $h \in \mathcal{C}\left(I,\left(\mathcal{N}_{A(t)}\right)_{t \in I}\right)$ and $\left(h_{k}\right)_{k \in \mathbf{N}}$ be a sequence such that $h_{k} \in \mathcal{C}\left(I,\left(\mathcal{N}_{A_{k}(t)}\right)_{t \in I}\right)$, for $k \in \mathbf{N}$, and

$$
\int_{T_{0}}^{t} h_{k}\left(v\left(\cdot, T_{0}, \tau ; h\right), \tau\right) d \tau \rightarrow \int_{T_{0}}^{t} h\left(v\left(\cdot, T_{0}, \tau ; h\right), \tau\right) d \tau, \quad \text { as } k \rightarrow \infty,
$$

locally uniformly on $\mathbf{B}^{n}$, for all $t \in I$. Then

$$
v\left(\cdot, T_{0}, t ; h_{k}\right) \rightarrow v\left(\cdot, T_{0}, t ; h\right), \quad \text { as } k \rightarrow \infty,
$$

locally uniformly on $\mathbf{B}^{n}$, for all $t \in I$. 
Remark 2.22. Recently, the authors [22] proved that if $T_{0} \geq 0$ and $A \in \widetilde{\mathcal{A}}$, then $\widetilde{\mathcal{R}}_{T}\left(\operatorname{id}_{\mathbf{B}^{n}},\left(\mathcal{N}_{A(t)}\right)_{t \in\left[T_{0}, T\right]}\right)$ is a compact family, for all $T>T_{0}$. Moreover, the family $\widetilde{\mathcal{R}}_{\infty}\left(\operatorname{id}_{\mathbf{B}^{n}},\left(\mathcal{N}_{A(t)}\right)_{t \geq T_{0}}\right)$ is also compact.

Now, we give the definition of the Hausdorff metric on $H\left(\mathbf{B}^{n}\right)$ (cf. [30]).

Definition 2.23. Let $\delta$ be the well known metric on $H\left(\mathbf{B}^{n}\right)$ such that $\left(H\left(\mathbf{B}^{n}\right), \delta\right)$ is a Fréchet space with respect to the compact-open topology. For all nonempty subsets $V$ and $W$ of $H\left(\mathbf{B}^{n}\right)$, let

$$
\delta(V, W)=\sup _{f \in V} \inf _{g \in W} \delta(f, g) .
$$

Also, let $\rho$ be the Hausdorff metric on $H\left(\mathbf{B}^{n}\right)$ given by

$$
\rho(V, W)=\max \{\delta(V, W), \delta(W, V)\},
$$

for all nonempty compact subsets $V$ and $W$ of $H\left(\mathbf{B}^{n}\right)$.

We close this section with the notions of extreme/support points associated with compact subsets of $H\left(\mathbf{B}^{n}\right)$ (see e.g. [8], [30]).

Definition 2.24. Let $E \subseteq H\left(\mathbf{B}^{n}\right)$ be a nonempty compact set.

(i) A point $f \in E$ is called an extreme point of $E$ (denoted by $f \in$ ex $E$ ) if $f=\lambda g+(1-\lambda) h$, for some $\lambda \in(0,1), g, h \in E$, implies that $f \equiv g \equiv h$.

(ii) A point $f \in E$ is called a support point of $E$ (denoted by $f \in \operatorname{supp} E$ ) if there exists a continuous linear functional $L: H\left(\mathbf{B}^{n}\right) \rightarrow \mathbf{C}$ such that $\Re L$ is nonconstant on $E$ and $\Re L(f)=\max _{g \in E} \Re L(g)$.

Remark 2.25. Let $\mathbf{A} \in L\left(\mathbf{C}^{n}\right)$ be such that $k_{+}(\mathbf{A})<2 m(\mathbf{A})$. In view of [13, Theorem 2.15], the family $S_{\mathbf{A}}^{0}\left(\mathbf{B}^{n}\right)$ is compact. Thus ex $S_{\mathbf{A}}^{0}\left(\mathbf{B}^{n}\right) \neq \emptyset$ and $\operatorname{supp} S_{\mathbf{A}}^{0}\left(\mathbf{B}^{n}\right) \neq \emptyset$.

\section{Convergence results for $\widetilde{S}_{A}^{T}\left(\mathrm{~B}^{n}\right)$ and for reachable families generated by time-dependent operators}

In this section we consider a dependence of $\widetilde{S}_{A}^{T}\left(\mathbf{B}^{n}\right)$ on $A \in \widetilde{\mathcal{A}}$, where $T \geq 0$ (cf. [22, Proposition 3.15]; cf. [30] for $n=1$ ). Note that the following results may be seen as dominated convergence type theorems. In the next section we shall apply Theorem 3.2 to obtain other results which involve time-dependent operators that are step functions (cf. Propositions 4.1 and 4.3).

Theorem 3.1. Let $I$ be the interval $\left[T_{0}, T\right]$, where $T>T_{0} \geq 0$, and $A \in \widetilde{\mathcal{A}}$ be such that $\operatorname{ess}_{\inf } \operatorname{ifI} m(A(t))>0$. Also, let $M>0$ and let $\left(A_{k}\right)_{k \in \mathbf{N}}$ be a sequence in $\widetilde{\mathcal{A}}$ such that $\left\|A_{k}(t)\right\| \leq M$, for a.e. $t \in I$ and for all $k \in \mathbf{N}$. If

$$
A_{k}(t) \rightarrow A(t), \text { as } k \rightarrow \infty \text {, for a.e. } t \in I,
$$

then

$$
\rho\left(\widetilde{\mathcal{R}}_{T}\left(\operatorname{id}_{\mathbf{B}^{n}},\left(\mathcal{N}_{A_{k}(t)}\right)_{t \in I}\right), \widetilde{\mathcal{R}}_{T}\left(\operatorname{id}_{\mathbf{B}^{n}},\left(\mathcal{N}_{A(t)}\right)_{t \in I}\right)\right) \rightarrow 0, \text { as } k \rightarrow \infty .
$$

Proof. First, we prove that $\delta\left(\widetilde{\mathcal{R}}_{T}\left(\operatorname{id}_{\mathbf{B}^{n}},\left(\mathcal{N}_{A_{k}(t)}\right)_{t \in I}\right), \widetilde{\mathcal{R}}_{T}\left(\operatorname{id}_{\mathbf{B}^{n}},\left(\mathcal{N}_{A(t)}\right)_{t \in I}\right)\right) \rightarrow 0$, as $k \rightarrow \infty$. Suppose that there are $\varepsilon>0$ and a nondecreasing sequence of indices $\left(k_{m}\right)_{m \in \mathbf{N}}$ such that for every $m \in \mathbf{N}$ we have

$$
\delta\left(\widetilde{\mathcal{R}}_{T}\left(\operatorname{id}_{\mathbf{B}^{n}},\left(\mathcal{N}_{A_{k m}(t)}\right)_{t \in I}\right), \widetilde{\mathcal{R}}_{T}\left(\operatorname{id}_{\mathbf{B}^{n}},\left(\mathcal{N}_{A(t)}\right)_{t \in I}\right)\right)>\varepsilon
$$


i.e. for every $m \in \mathbf{N}$ there exists $f_{m} \in \widetilde{\mathcal{R}}_{T}\left(\operatorname{id}_{\mathbf{B}^{n}},\left(\mathcal{N}_{A_{k_{m}}(t)}\right)_{t \in I}\right)$ such that for every $g \in \widetilde{\mathcal{R}}_{T}\left(\operatorname{id}_{\mathbf{B}^{n}},\left(\mathcal{N}_{A(t)}\right)_{t \in I}\right)$ we have $\delta\left(f_{m}, g\right)>\varepsilon$.

Let $m \in \mathbf{N}$ be arbitrary. Since $f_{m} \in \widetilde{\mathcal{R}}_{T}\left(\operatorname{id}_{\mathbf{B}^{n}},\left(\mathcal{N}_{A_{k_{m}}(t)}\right)_{t \in I}\right)$, there exists $h_{m} \in$ $\mathcal{C}\left(I,\left(\mathcal{N}_{A_{k_{m}}(t)}\right)_{t \in I}\right)$ such that $f_{m}=V_{m}\left(T_{0}, T\right)^{-1} v\left(\cdot, T_{0}, T ; h_{m}\right)$, where $V_{m}\left(T_{0}, \cdot\right)$ is the unique solution on $\left[T_{0}, \infty\right)$ of the initial value problem (2.1) related to $A_{k_{m}}$. By [19, Lemma 3], for every $r \in(0,1)$ we have

$\Re\left\langle\frac{1}{r} h_{m}(r z, t)-\left(A_{k_{m}}(t) z-A(t) z\right), z\right\rangle \geq\left(m\left(A_{k_{m}}(t)\right) \frac{1-r}{1+r}-\left\|A_{k_{m}}(t)-A(t)\right\|\right)\|z\|^{2}$, $z \in \mathbf{B}^{n}, t \in I$. For every $l \in \mathbf{N}$, by Egorov's Theorem, there exists a measurable set $N_{l} \subset I$ such that $\lambda\left(N_{l}\right) \leq \frac{1}{l}$ and $\left(A_{k_{m}}\right)_{m \in \mathbf{N}}$ converges to $A$ uniformly on $I \backslash N_{l}$. Since essinf $\inf _{t \in I} m(A(t))>0$, we deduce that for every $l \in \mathbf{N}$ there is $m_{l} \in \mathbf{N}$ such that $q_{l}: \mathbf{B}^{n} \times I \rightarrow \mathbf{C}^{n}$ given by

$$
q_{l}(z, t)=\left\{\begin{array}{ll}
\frac{1}{r_{l}} h_{m_{l}}\left(r_{l} z, t\right)-A_{k_{m_{l}}}(t) z+A(t) z, & t \in I \backslash N_{l} \\
A(t) z, & t \in N_{l}
\end{array},\right.
$$

for all $z \in \mathbf{B}^{n}$, satisfies $q_{l} \in \mathcal{C}\left(I,\left(\mathcal{N}_{A(t)}\right)_{t \in I}\right)$, where $r_{l}=1-\frac{1}{l}$.

For every $l \in \mathbf{N}$ and $t \in I$ the following equality holds:

$$
\frac{1}{r_{l}} h_{m_{l}}\left(r_{l} z, t\right)-h_{m_{l}}(z, t)=\frac{r_{l}-1}{r_{l}} \int_{0}^{1}\left(D h_{m_{l}}\left(\tau r_{l} z+(1-\tau) z, t\right)(z)-h_{m_{l}}(z, t)\right) d \tau \text {. }
$$

Since $\left\|A_{k_{m_{l}}}(t)\right\| \leq M$, for a.e. $t \in I$ and for all $l \in \mathbf{N}$, we deduce in view Lemma 2.1 that there is a null set $J \subseteq I$ such that $\left\{h_{m_{l}}(\cdot, t)\right\}_{t \in I \backslash J, l \in \mathbf{N}}$ is a normal family. Hence, using (3.1) and the fact that $r_{l} \rightarrow 1$ as $l \rightarrow \infty$, we obtain for a.e. $t \in I$ that

$$
q_{l}(\cdot, t)-h_{m_{l}}(\cdot, t) \rightarrow 0, \text { as } l \rightarrow \infty \text {, locally uniformly on } \mathbf{B}^{n} \text {. }
$$

Using Lemma 2.20, we deduce that there is $q \in \mathcal{C}\left(I,\left(\mathcal{N}_{A(t)}\right)_{t \in I}\right)$ such that up to a subsequence, we have

$$
\int_{T_{0}}^{t} q_{l}\left(v\left(\cdot, T_{0}, \tau ; q\right), \tau\right) d \tau \rightarrow \int_{T_{0}}^{t} q\left(v\left(\cdot, T_{0}, \tau ; q\right), \tau\right) d \tau, \quad \text { as } l \rightarrow \infty
$$

locally uniformly on $\mathbf{B}^{n}$, for all $t \in I$. Since $\operatorname{ess}_{\sup }\|t\| A(t) \|<\infty$, we deduce by Lemma 2.1 that there is a null set $J^{\prime} \subseteq I$ such that $\left\{q_{l}(\cdot, t)\right\}_{t \in I \backslash J^{\prime}, l \in \mathbf{N}}$ is a normal family. Hence, in view of (3.2), (3.3) and the Lebesgue dominated convergence theorem, we obtain that

$$
\int_{T_{0}}^{t} h_{m_{l}}\left(v\left(\cdot, T_{0}, \tau ; q\right), \tau\right) d \tau \rightarrow \int_{T_{0}}^{t} q\left(v\left(\cdot, T_{0}, \tau ; q\right), \tau\right) d \tau, \text { as } l \rightarrow \infty
$$

locally uniformly on $\mathbf{B}^{n}$, for all $t \in I$. In view of (3.3) and (3.4), we apply Lemma 2.21 to deduce that

$$
v\left(\cdot, T_{0}, T ; h_{m_{l}}\right) \rightarrow v\left(\cdot, T_{0}, T ; q\right) \text { and } v\left(\cdot, T_{0}, T ; q_{l}\right) \rightarrow v\left(\cdot, T_{0}, T ; q\right) \text {, as } l \rightarrow \infty
$$

locally uniformly on $\mathbf{B}^{n}$.

Let $V(s, t)$ be the unique solution on $[s, \infty)$ of the initial value problem $(2.1)$ related to $A$. In view of (3.5) and Weierstrass' convergence theorem, we deduce that $D v\left(0, T_{0}, T ; h_{m_{l}}\right) \rightarrow D v\left(0, T_{0}, T ; q\right)$, as $l \rightarrow \infty$. Since $D v\left(0, T_{0}, T ; h_{m_{l}}\right)=V_{m_{l}}\left(T_{0}, T\right)$, for $l \in \mathbf{N}$, and $D v\left(0, T_{0}, T ; q\right)=V\left(T_{0}, T\right)$ (see [22]), it follows that $V_{m_{l}}\left(T_{0}, T\right) \rightarrow$ $V\left(T_{0}, T\right)$, as $l \rightarrow \infty$. Since $V\left(T_{0}, T\right)$ and $V_{m_{l}}\left(T_{0}, T\right)$, for $l \in \mathbf{N}$, are invertible operators, it is easy to prove that (cf. [9, Theorem 2.17])

$$
V_{m_{l}}\left(T_{0}, T\right)^{-1} \rightarrow V\left(T_{0}, T\right)^{-1}, \text { as } l \rightarrow \infty
$$


Let $g=V\left(T_{0}, T\right)^{-1} v\left(\cdot, T_{0}, T ; q\right)$ and $g_{l}=V\left(T_{0}, T\right)^{-1} v\left(\cdot, T_{0}, T ; q_{l}\right)$, for $l \in \mathbf{N}$. In view of (3.5) and (3.6), we deduce that $g_{l} \rightarrow g$ and $f_{m_{l}} \rightarrow g$ locally uniformly on $\mathbf{B}^{n}$, as $l \rightarrow \infty$. Hence $\delta\left(f_{m_{l}}, g_{l}\right) \rightarrow 0$, as $l \rightarrow \infty$. However, this is a contradiction, since $g_{l} \in \widetilde{\mathcal{R}}_{T}\left(\operatorname{id}_{\mathbf{B}^{n}},\left(\mathcal{N}_{A(t)}\right)_{t \in I}\right)$, for all $l \in \mathbf{N}$.

To prove that $\delta\left(\widetilde{\mathcal{R}}_{T}\left(\operatorname{id}_{\mathbf{B}^{n}},\left(\mathcal{N}_{A(t)}\right)_{t \in I}\right), \widetilde{\mathcal{R}}_{T}\left(\operatorname{id}_{\mathbf{B}^{n}},\left(\mathcal{N}_{A_{k}(t)}\right)_{t \in I}\right)\right) \rightarrow 0$, as $k \rightarrow \infty$, it suffices to use similar arguments as before. This completes the proof.

Theorem 3.2. Let $T \geq 0$ and $A \in \widetilde{\mathcal{A}}$ be such that $\operatorname{essinf}_{t \geq T} m(A(t))>0$. Also, let $M>0, \alpha \in L^{1}([T, \infty), \mathbf{R})$ and $\left(A_{k}\right)_{k \in \mathbf{N}}$ be a sequence in $\widetilde{\mathcal{A}}$ such that $\left\|A_{k}(t)\right\| \leq M$ and $\left\|V_{k}(T, t)^{-1}\right\| e^{-2 \int_{T}^{t} m\left(A_{k}(\tau)\right) d \tau} \leq \alpha(t)$, for a.e. $t \geq T$ and for all $k \in \mathbf{N}$, where $V_{k}(T, \cdot)$ is the unique solution on $[T, \infty)$ of the initial value problem (2.1) related to $A_{k}$. If

$$
A_{k}(t) \rightarrow A(t), \text { as } k \rightarrow \infty, \text { for a.e. } t \geq T
$$

then

$$
\rho\left(\widetilde{S}_{A_{k}}^{T}\left(\mathbf{B}^{n}\right), \widetilde{S}_{A}^{T}\left(\mathbf{B}^{n}\right)\right) \rightarrow 0, \text { as } k \rightarrow \infty .
$$

Proof. First, we prove that for every sequence $\left(T_{k}\right)_{k \in \mathbf{N}}$ in $(T, \infty)$ such that $T_{k} \rightarrow \infty$, as $k \rightarrow \infty$, we have

$$
\rho\left(\widetilde{\mathcal{R}}_{T_{k}}\left(\operatorname{id}_{\mathbf{B}^{n}},\left(\mathcal{N}_{A_{k}(t)}\right)_{t \in\left[T, T_{k}\right]}\right), \widetilde{S}_{A_{k}}^{T}\left(\mathbf{B}^{n}\right)\right) \rightarrow 0, \text { as } k \rightarrow \infty .
$$

Fix a sequence $\left(T_{k}\right)_{k \in \mathbf{N}}$ in $(T, \infty)$ such that $T_{k} \rightarrow \infty$, as $k \rightarrow \infty$. Let $k \in \mathbf{N}$ and $f_{k} \in \widetilde{S}_{A_{k}}^{T}\left(\mathbf{B}^{n}\right)$ be arbitrary. Then there is $h_{k} \in \mathcal{C}\left([T, \infty),\left(\mathcal{N}_{A_{k}(t)}\right)_{t \geq T}\right)$ such that $f_{k}=\lim _{t \rightarrow \infty} V_{k}(T, t)^{-1} v\left(\cdot, T, t ; h_{k}\right)$. Let $g_{k}=V_{k}\left(T, T_{k}\right)^{-1} v\left(\cdot, T, T_{k} ; h_{k}\right)$. Then $g_{k} \in \widetilde{\mathcal{R}}_{T_{k}}\left(\operatorname{id}_{\mathbf{B}^{n}},\left(\mathcal{N}_{A_{k}(t)}\right)_{t \in\left[T, T_{k}\right]}\right)$. Taking into account Lemma 2.15, we deduce that for every $r \in(0,1)$ there is $C_{r}>0$ such that

$$
\left\|f_{k}-g_{k}\right\|_{\overline{\mathbf{B}_{r}^{n}}} \leq C_{r} \int_{T_{k}}^{\infty}\left\|A_{k}(t)\right\|\left\|V_{k}(T, t)^{-1}\right\| e^{-2 \int_{T}^{t} m\left(A_{k}(\tau)\right) d \tau} d t \leq C_{r} M \int_{T_{k}}^{\infty} \alpha(t) d t .
$$

Hence

$$
\delta\left(\widetilde{S}_{A_{k}}^{T}\left(\mathbf{B}^{n}\right), \widetilde{\mathcal{R}}_{T_{k}}\left(\operatorname{id}_{\mathbf{B}^{n}},\left(\mathcal{N}_{A_{k}(t)}\right)_{t \in\left[T, T_{k}\right]}\right)\right) \rightarrow 0, \text { as } k \rightarrow \infty .
$$

By Remark 2.19

$$
\delta\left(\widetilde{\mathcal{R}}_{T_{k}}\left(\operatorname{id}_{\mathbf{B}^{n}},\left(\mathcal{N}_{A_{k}(t)}\right)_{t \in\left[T, T_{k}\right]}\right), \widetilde{S}_{A_{k}}^{T}\left(\mathbf{B}^{n}\right)\right)=0, \text { for all } k \in \mathbf{N},
$$

and thus we obtain (3.7).

In the same manner, since $A \in \widetilde{\mathcal{A}}$, we can also prove that for every sequence $\left(T_{k}\right)_{k \in \mathbf{N}}$ in $(T, \infty)$ such that $T_{k} \rightarrow \infty$, as $k \rightarrow \infty$, we have

$$
\rho\left(\widetilde{\mathcal{R}}_{T_{k}}\left(\operatorname{id}_{\mathbf{B}^{n}},\left(\mathcal{N}_{A(t)}\right)_{t \in\left[T, T_{k}\right]}\right), \widetilde{S}_{A}^{T}\left(\mathbf{B}^{n}\right)\right) \rightarrow 0, \text { as } k \rightarrow \infty .
$$

Let $\left(A_{k_{m}}\right)_{m \in \mathbf{N}}$ be an arbitrary subsequence of $\left(A_{k}\right)_{k \in \mathbf{N}}$. Let $\left(T_{l}\right)_{l \in \mathbf{N}}$ be a sequence in $(T, \infty)$ such that $T_{l} \rightarrow \infty$, as $l \rightarrow \infty$. By Theorem 3.1, we deduce that for every $l \in \mathbf{N}$ there is $m_{l} \in \mathbf{N}$ such that

$$
\rho\left(\widetilde{\mathcal{R}}_{T_{l}}\left(\operatorname{id}_{\mathbf{B}^{n}},\left(\mathcal{N}_{A_{k_{m}}}(t)\right)_{t \in\left[T, T_{l}\right]}\right), \widetilde{\mathcal{R}}_{T_{l}}\left(\operatorname{id}_{\mathbf{B}^{n}},\left(\mathcal{N}_{A(t)}\right)_{t \in\left[T, T_{l}\right]}\right)\right) \leq \frac{1}{l} .
$$

Taking into account (3.7) and (3.8), we deduce that

$$
\rho\left(\widetilde{S}_{A_{k_{m}}}^{T}\left(\mathbf{B}^{n}\right), \widetilde{S}_{A}^{T}\left(\mathbf{B}^{n}\right)\right) \rightarrow 0, \text { as } l \rightarrow \infty .
$$

Since every subsequence of $\left(\widetilde{S}_{A_{k}}^{T}\left(\mathbf{B}^{n}\right)\right)_{k \in \mathbf{N}}$ contains a subsequence that converges to $\widetilde{S}_{A}^{T}\left(\mathbf{B}^{n}\right)$, we deduce that $\left(\widetilde{S}_{A_{k}}^{T}\left(\mathbf{B}^{n}\right)\right)_{k \in \mathbf{N}}$ converges to $\widetilde{S}_{A}^{T}\left(\mathbf{B}^{n}\right)$. 
Corollary 3.3. Let $T \geq 0$ and $A \in \widetilde{\mathcal{A}}$ be such that $\operatorname{ess~} \inf _{t \geq T} m(A(t))>0$. Also, let $T^{\prime}>T, M>0$ and $\left(A_{k}\right)_{k \in \mathbf{N}}$ be a sequence in $\widetilde{\mathcal{A}}$ such that $\left\|A_{k}(t)\right\| \leq M$, for a.e. $t \in\left[T, T^{\prime}\right]$, and $A_{k}(t)=A(t)$, for a.e. $t>T^{\prime}$, and for all $k \in \mathbf{N}$. If

$$
A_{k}(t) \rightarrow A(t), \quad \text { as } k \rightarrow \infty, \text { for a.e. } t \in\left[T, T^{\prime}\right],
$$

then

$$
\rho\left(\widetilde{S}_{A_{k}}^{T}\left(\mathbf{B}^{n}\right), \widetilde{S}_{A}^{T}\left(\mathbf{B}^{n}\right)\right) \rightarrow 0, \text { as } k \rightarrow \infty .
$$

Proof. Let $V(T, \cdot)$ be the unique solution on $[T, \infty)$ of the initial value problem (2.1) related to $A$ and for every $k \in \mathbf{N}$ let $V_{k}(T, \cdot)$ be the unique solution on $[T, \infty)$ of the initial value problem (2.1) related to $A_{k}$. For every $k \in \mathbf{N}$ we have (see Lemma 2.3)

$$
\begin{aligned}
& \left\|V_{k}(T, t)^{-1}\right\| e^{-2 \int_{T}^{t} m\left(A_{k}(\tau)\right) d \tau} \\
& \leq\left\|V_{k}\left(T, T^{\prime}\right)^{-1}\right\| e^{-2 \int_{T}^{T^{\prime}} m\left(A_{k}(\tau)\right) d \tau}\left\|V\left(T^{\prime}, t\right)^{-1}\right\| e^{-2 \int_{T^{\prime}}^{t} m(A(\tau)) d \tau} \\
& \leq e^{\left(T^{\prime}-T\right) M}\left\|V\left(T^{\prime}, t\right)^{-1}\right\| e^{-2 \int_{T^{\prime}}^{t} m(A(\tau)) d \tau}
\end{aligned}
$$

for all $t \geq T^{\prime}$. Let $\alpha:[T, \infty) \rightarrow \mathbf{R}$ be given by

$$
\alpha(t)=\left\{\begin{array}{ll}
e^{(t-T) M}, & t \in\left[T, T^{\prime}\right) \\
e^{\left(T^{\prime}-T\right) M}\left\|V\left(T^{\prime}, t\right)^{-1}\right\| e^{-2 \int_{T^{\prime}}^{t} m(A(\tau)) d \tau}, & t \in\left[T^{\prime}, \infty\right)
\end{array} .\right.
$$

Since $A \in \widetilde{\mathcal{A}}$, we have $\alpha \in L^{1}([T, \infty), \mathbf{R})$. By Theorem 3.2, the proof is done.

For constant time-dependent operators (cf. Remark 2.11), we have the following result.

Theorem 3.4. Let $\mathbf{A} \in L\left(\mathbf{C}^{n}\right)$ be such that $k_{+}(\mathbf{A})<2 m(\mathbf{A})$, and let $\left(\mathbf{A}_{l}\right)_{l \in \mathbf{N}}$ be a sequence in $L\left(\mathbf{C}^{n}\right)$ such that $\mathbf{A}_{l} \rightarrow \mathbf{A}$, as $l \rightarrow \infty$. Then there is $l_{0} \in \mathbf{N}$ such that $S_{\mathbf{A}_{l}}^{0}\left(\mathbf{B}^{n}\right)$ is compact for $l \geq l_{0}$, and $\rho\left(S_{\mathbf{A}_{l}}^{0}\left(\mathbf{B}^{n}\right), S_{\mathbf{A}}^{0}\left(\mathbf{B}^{n}\right)\right) \rightarrow 0$, as $l \rightarrow \infty$.

Proof. First, we observe that for every $l \in \mathbf{N}$ we have

$$
m\left(\mathbf{A}_{l}\right)-\left\|\mathbf{A}-\mathbf{A}_{l}\right\| \leq m(\mathbf{A}) \leq m\left(\mathbf{A}_{l}\right)+\left\|\mathbf{A}-\mathbf{A}_{l}\right\| .
$$

Hence

$$
\limsup _{l \rightarrow \infty} m\left(\mathbf{A}_{l}\right) \leq m(\mathbf{A}) \leq \liminf _{l \rightarrow \infty} m\left(\mathbf{A}_{l}\right)
$$

and thus $\lim _{l \rightarrow \infty} m\left(\mathbf{A}_{l}\right)=m(\mathbf{A})$. that

$$
\left\|e^{t \mathbf{A}}\right\| \leq \delta e^{(2 m(\mathbf{A})-\varepsilon / 2) t}, \quad t \geq 0 .
$$

Let $l_{0} \in \mathbf{N}$ be such that for every $l \geq l_{0}$ we have

$$
2 m(\mathbf{A})-2 m\left(\mathbf{A}_{l}\right)+\delta\left\|\mathbf{A}-\mathbf{A}_{l}\right\| \leq \varepsilon / 4 .
$$

Taking into account the proof of [26, Theorem 2.1, pp. 497-498], and using (3.9) and (3.10), we deduce that for every $l \geq l_{0}$ we have

$$
\left\|e^{t \mathbf{A}_{l}}\right\| \leq \delta e^{\left(2 m(\mathbf{A})-\varepsilon / 2+\delta\left\|\mathbf{A}_{l}-\mathbf{A}\right\|\right) t} \leq \delta e^{-t \varepsilon / 4} e^{2 m\left(\mathbf{A}_{l}\right) t}, \text { for all } t \geq 0 .
$$

Let $\alpha:[0, \infty) \rightarrow \mathbf{R}$ be given by $\alpha(t)=\delta e^{-t \varepsilon / 4}$, for all $t \geq 0$. Then $\alpha \in$ $L^{1}([0, \infty), \mathbf{R})$ and $\left\|e^{t \mathbf{A}_{l}}\right\| e^{-2 m\left(\mathbf{A}_{l}\right) t} \leq \alpha(t)$, for all $t \geq 0$ and $l \geq \overline{l_{0}}$. So, by $[9$, Remark 2.8], we have that $k_{+}\left(\mathbf{A}_{l}\right)<2 m\left(\mathbf{A}_{l}\right)$, and thus $S_{\mathbf{A}_{l}}^{0}\left(\mathbf{B}^{n}\right)$ is compact, for all 
$l \geq l_{0}$. Moreover, by Theorem 3.2, we have $\rho\left(S_{\mathbf{A}_{l}}^{0}\left(\mathbf{B}^{n}\right), S_{\mathbf{A}}^{0}\left(\mathbf{B}^{n}\right)\right) \rightarrow 0$, as $l \rightarrow \infty$. This completes the proof.

Taking into account Theorem 3.4, it is natural to ask the following question.

Question 3.5. Under the assumptions of Theorem 3.4, is it true that

$$
\lim _{l \rightarrow \infty} \rho\left(\overline{\operatorname{ex} S_{\mathbf{A}_{l}}^{0}\left(\mathbf{B}^{n}\right)}, \overline{\operatorname{ex} S_{\mathbf{A}}^{0}\left(\mathbf{B}^{n}\right)}\right)=0 \text { and } \lim _{l \rightarrow \infty} \rho\left(\overline{\operatorname{supp} S_{\mathbf{A}_{l}}^{0}\left(\mathbf{B}^{n}\right)}, \overline{\operatorname{supp} S_{\mathbf{A}}^{0}\left(\mathbf{B}^{n}\right)}\right)=0 ?
$$

In view of Theorem 3.4, we obtain the following convergence result related to the Carathéodory family $\mathcal{N}_{\mathbf{A}}$, where $\mathbf{A} \in L\left(\mathbf{C}^{n}\right)$ with $m(\mathbf{A})>0$. This result is motivated by the fact that every mapping $f \in S_{\mathbf{A}}^{0}\left(\mathbf{B}^{n}\right)$ is generated by a Herglotz vector field $h: \mathbf{B}^{n} \times[0, \infty) \rightarrow \mathbf{C}^{n}$ with respect to $\mathbf{A}$.

Proposition 3.6. Let $\mathbf{A} \in L\left(\mathbf{C}^{n}\right)$ be such that $m(\mathbf{A})>0$, and let $\left(\mathbf{A}_{k}\right)_{k \in \mathbf{N}}$ be a sequence in $L\left(\mathbf{C}^{n}\right)$ such that $\mathbf{A}_{k} \rightarrow \mathbf{A}$, as $k \rightarrow \infty$. Then there is $k_{0} \in \mathbf{N}$ such that $m\left(\mathbf{A}_{k}\right)>0$, for all $k \geq k_{0}$, and $\rho\left(\mathcal{N}_{\mathbf{A}_{k}}, \mathcal{N}_{\mathbf{A}}\right) \rightarrow 0$, as $k \rightarrow \infty$.

Proof. Since $\lim _{k \rightarrow \infty} m\left(\mathbf{A}_{k}\right)=m(\mathbf{A})$ by the proof of Theorem 3.4, and since $m(\mathbf{A})>0$, it follows that there is $k_{0} \in \mathbf{N}$ such that $m\left(\mathbf{A}_{k}\right)>0$, for all $k \geq k_{0}$. Hence, $\mathcal{N}_{\mathbf{A}_{k}}$ is well defined, for all $k \geq k_{0}$.

The fact that $\rho\left(\mathcal{N}_{\mathbf{A}_{k}}, \mathcal{N}_{\mathbf{A}}\right) \rightarrow 0$, as $k \rightarrow \infty$, follows by arguments similar to those in the proof of Theorem 3.1. Indeed, suppose that there exist $\varepsilon>0$, a sequence of indices $\left(k_{m}\right)_{m \in \mathbf{N}}$ with $k_{m} \geq k_{0}, m \in \mathbf{N}$, and a sequence of mappings $\left(h_{m}\right)_{m \in \mathbf{N}}$ with $h_{m} \in \mathcal{N}_{\mathbf{A}_{k_{m}}}, m \in \mathbf{N}$, such that $\delta\left(h_{m}, h\right)>\varepsilon$, for all $h \in \mathcal{N}_{\mathbf{A}}$. In view of [19, Lemma 3], we deduce that for every $l \in \mathbf{N}$, there exists $m_{l} \in \mathbf{N}$ such that $q_{l} \in \mathcal{N}_{\mathbf{A}}$, where $q_{l}(z)=\frac{1}{r_{l}} h_{m_{l}}\left(r_{l} z\right)-\mathbf{A}_{k_{m_{l}}} z+\mathbf{A} z$, for all $z \in \mathbf{B}^{n}$, and $r_{l}=1-\frac{1}{l}$. Since $\mathbf{A}_{k_{m_{l}}} \rightarrow \mathbf{A}$, as $l \rightarrow \infty$, we deduce that there is $M>0$ such that $\left\|\mathbf{A}_{k_{m_{l}}}\right\| \leq M$, for all $l \in \mathbf{N}$. Hence, as in the proof of Theorem 3.1, we get that $h_{m_{l}}-q_{l} \rightarrow 0$, as $l \rightarrow \infty$, locally uniformly on $\mathbf{B}^{n}$, which is a contradiction. Thus, $\delta\left(\mathcal{N}_{\mathbf{A}_{k}}, \mathcal{N}_{\mathbf{A}}\right) \rightarrow 0$, as $k \rightarrow \infty$. The fact that $\delta\left(\mathcal{N}_{\mathbf{A}}, \mathcal{N}_{\mathbf{A}_{k}}\right) \rightarrow 0$, as $k \rightarrow \infty$, follows by the same arguments as above.

We close this section with the following convergence result for the family $\widehat{S}_{\mathbf{A}}\left(\mathbf{B}^{n}\right)$ of spirallike mappings with respect to $\mathbf{A} \in L\left(\mathbf{C}^{n}\right)$, where $k_{+}(\mathbf{A})<2 m(\mathbf{A})$.

Proposition 3.7. Let $\mathbf{A} \in L\left(\mathbf{C}^{n}\right)$ be such that $k_{+}(\mathbf{A})<2 m(\mathbf{A})$, and let $\left(\mathbf{A}_{l}\right)_{l \in \mathbf{N}}$ be a sequence in $L\left(\mathbf{C}^{n}\right)$ such that $\mathbf{A}_{l} \rightarrow \mathbf{A}$, as $l \rightarrow \infty$. Then there is $l_{0} \in \mathbf{N}$ such that $\widehat{S}_{\mathbf{A}_{l}}\left(\mathbf{B}^{n}\right)$ is compact for $l \geq l_{0}$, and $\rho\left(\widehat{S}_{\mathbf{A}_{l}}\left(\mathbf{B}^{n}\right), \widehat{S}_{\mathbf{A}}\left(\mathbf{B}^{n}\right)\right) \rightarrow 0$, as $l \rightarrow \infty$.

Proof. By the proof of Theorem 3.4, we have that there exist $l_{0} \in \mathbf{N}$ and $\alpha \in L^{1}([0, \infty), \mathbf{R})$ such that $\left\|e^{t \mathbf{A}_{l}}\right\| e^{-2 m\left(\mathbf{A}_{l}\right) t} \leq \alpha(t)$, for all $t \geq 0$ and $l \geq l_{0}$. In particular, $k_{+}\left(\mathbf{A}_{l}\right)<2 m\left(\mathbf{A}_{l}\right)$, by [9, Remark 2.8], and thus $\widehat{S}_{\mathbf{A}_{l}}\left(\mathbf{B}^{n}\right)$ is compact, for all $l \geq l_{0}$, by [35, Theorem 3.1] (cf. [13]).

Finally, since every spirallike mapping is generated by a Herglotz vector field that is constant in time (see [13]; cf. [10]), we may adapt all arguments in the proof of Theorems 3.1 and 3.2 and deduce that $\lim _{l \rightarrow \infty} \rho\left(\widehat{S}_{\mathbf{A}_{l}}\left(\mathbf{B}^{n}\right), \widehat{S}_{\mathbf{A}}\left(\mathbf{B}^{n}\right)\right)=0$, as desired.

Question 3.8. In connection with [1] and [35], would be possible to generalize Theorem 3.4 and Proposition 3.7 to the case of non-resonant linear operators? 


\section{Analytical characterizations of mappings in $\widetilde{S}_{A}^{t}\left(\mathrm{~B}^{n}\right)$}

In this section we obtain some sufficient conditions related to $A \in \widetilde{\mathcal{A}}$, which guarantee the equality $\widetilde{S}_{A}^{t}\left(\mathbf{B}^{n}\right)=S^{0}\left(\mathbf{B}^{n}\right)$, for $t \geq 0$. The first result is a generalization of [13, Theorem 3.12].

Proposition 4.1. Let $k \in \mathbf{N}, \alpha_{1}, \ldots, \alpha_{k}>0$, and let $E_{1}, \ldots, E_{k} \in L\left(\mathbf{C}^{n}\right)$ be such that $E_{i}+E_{i}^{*}=2 \alpha_{i} I_{n}$, for all $i \in\{1, \ldots, k\}$. Also, let $0=T_{0}<T_{1}<\ldots<$ $T_{k-1}<T_{k}=\infty$ and let $A:[0, \infty) \rightarrow L\left(\mathbf{C}^{n}\right)$ be given by

$$
A(t)= \begin{cases}E_{1}, & \text { for } t \in\left[T_{0}, T_{1}\right), \\ \vdots & \\ E_{k}, & \text { for } t \in\left[T_{k-1}, T_{k}\right) .\end{cases}
$$

Then $\widetilde{S}_{A}^{T}\left(\mathbf{B}^{n}\right)=S^{0}\left(\mathbf{B}^{n}\right)$, for all $T \geq 0$.

Proof. We shall use arguments similar to those in the proof of [13, Theorem 3.12]. Fix $T \geq 0$ and let $i \in\{1, \ldots, k\}$ be such that $T \in\left[T_{i-1}, T_{i}\right)$.

First, we prove that $S^{0}\left(\mathbf{B}^{n}\right) \subseteq \widetilde{S}_{A}^{T}\left(\mathbf{B}^{n}\right)$. To this end, let $f \in S^{0}\left(\mathbf{B}^{n}\right)$. Then there exists a Herglotz vector field $h: \mathbf{B}^{n} \times[0, \infty) \rightarrow \mathbf{C}^{n}\left(\right.$ with $D h(0, \cdot) \equiv I_{n}$ ) such that $f=\lim _{t \rightarrow \infty} e^{t} v(\cdot, 0, t)$ locally uniformly on $\mathbf{B}^{n}$, where $v(z, 0, \cdot)$ is the unique locally absolutely continuous solution of the initial value problem

$$
\frac{\partial v}{\partial t}(z, 0, t)=-h(v(z, 0, t), t), \text { a.e. } t \in[0, \infty), v(z, 0,0)=z,
$$

for all $z \in \mathbf{B}^{n}$. For each $j \in\{i, \ldots, k\}$, let $F_{j}:[0, \infty) \rightarrow L\left(\mathbf{C}^{n}\right)$ be recursively given by

and

$$
F_{i}(t)=e^{(t-T)\left(\alpha_{i} I_{n}-E_{i}\right)}, \quad t \geq 0,
$$

$$
F_{j}(t)=e^{\left(t-T_{j-1}\right)\left(\alpha_{j} I_{n}-E_{j}\right)} F_{j-1}\left(T_{j-1}\right), \quad j \neq i, t \geq 0 .
$$

Also, let $\beta_{j}:[0, \infty) \rightarrow \mathbf{R}$ be given

$$
\beta_{i}(t)=\alpha_{i}(t-T) \text { and } \beta_{j}(t)=\alpha_{j}\left(t-T_{j-1}\right)+\beta_{j-1}\left(T_{j-1}\right), \quad j \neq i, t \geq 0 .
$$

Let $q: \mathbf{B}^{n} \times[0, \infty) \rightarrow \mathbf{C}^{n}$ be given by

$$
q(z, t)= \begin{cases}A(t) z, & t \in[0, T), \\ \alpha_{i} F_{i}(t) h\left(F_{i}(t)^{-1} z, \beta_{i}(t)\right)-\left(\alpha_{i} I_{n}-E_{i}\right) z, & t \in\left[T, T_{i}\right), \\ \alpha_{i+1} F_{i+1}(t) h\left(F_{i+1}(t)^{-1} z, \beta_{i+1}(t)\right)-\left(\alpha_{i+1} I_{n}-E_{i+1}\right) z, & t \in\left[T_{i}, T_{i+1}\right), \\ \vdots & t \in\left[T_{k-1}, T_{k}\right), \\ \alpha_{k} F_{k}(t) h\left(F_{k}(t)^{-1} z, \beta_{k}(t)\right)-\left(\alpha_{k} I_{n}-E_{k}\right) z, & \end{cases}
$$

for all $z \in \mathbf{B}^{n}$. Since $E_{j}+E_{j}^{*}=2 \alpha_{j} I_{n}$, for all $j \in\{i, \ldots, k\}$, we deduce by an inductive argument that $F_{j}(t)^{*}=F_{j}(t)^{-1}$ and $\left\|F_{j}(t)^{-1}\right\| \leq 1$, for all $t \in\left[T_{j-1}, T_{j}\right)$ and $j \in\{i, \ldots, k\}$ (cf. [13]). Then it is not difficult to prove that $q$ is well defined and is a Herglotz vector field with respect to $A$.

Let

$$
u(z, T, t)= \begin{cases}F_{i}(t) v\left(z, 0, \beta_{i}(t)\right), & \text { for } z \in \mathbf{B}^{n}, t \in\left[T, T_{i}\right), \\ F_{i+1}(t) v\left(z, 0, \beta_{i+1}(t)\right), & \text { for } z \in \mathbf{B}^{n}, t \in\left[T_{i}, T_{i+1}\right), \\ \vdots & \text { for } z \in \mathbf{B}^{n}, t \in\left[T_{k-1}, T_{k}\right) . \\ F_{k}(t) v\left(z, 0, \beta_{k}(t)\right), & \end{cases}
$$


We observe that $\frac{d}{d t} F_{j}(t)=\left(\alpha_{j} I_{n}-E_{j}\right) F_{j}(t)$, for all $j \in\{i, \ldots, k\}$ and every $t \in$ $\left[T_{j-1}, T_{j}\right)$, and thus $u(z, T, \cdot)$ is the unique locally absolutely continuous solution of the initial value problem

$$
\frac{\partial u}{\partial t}(z, T, t)=-q(u(z, T, t), t), \quad \text { a.e. } t \in[T, \infty), u(z, T, T)=z,
$$

for all $z \in \mathbf{B}^{n}$.

Let $V(T, \cdot)$ be the unique solution on $[T, \infty)$ of the initial value problem $(2.1)$ related to $A$. Since $V(T, t)=e^{-\beta_{k}(t)} F_{k}(t)$, for all $t \geq T_{k-1}$, and $\lim _{t \rightarrow \infty} \beta_{k}(t)=$ $\infty$, we deduce that $f=\lim _{t \rightarrow \infty} e^{\beta_{k}(t)} v\left(\cdot, 0, \beta_{k}(t)\right)=\lim _{t \rightarrow \infty} V(T, t)^{-1} u(\cdot, T, t)$ locally uniformly on $\mathbf{B}^{n}$. Hence $f \in \widetilde{S}_{A}^{T}\left(\mathbf{B}^{n}\right)$. So $S^{0}\left(\mathbf{B}^{n}\right) \subseteq \widetilde{S}_{A}^{T}\left(\mathbf{B}^{n}\right)$.

Using similar arguments as above, we may prove that $\widetilde{S}_{A}^{T}\left(\mathbf{B}^{n}\right) \subseteq S^{0}\left(\mathbf{B}^{n}\right)$ (cf. [13, Remark 3.13]). This completes the proof.

In view of Propositions 2.13 and 4.1, we obtain the following example.

Example 4.2. Let $E=\left(\begin{array}{cc}1 & i \\ i & 1\end{array}\right)$ and $T>0$. Let $A \in \widetilde{\mathcal{A}}$ be given by

$$
A(t)= \begin{cases}E, & \text { for } t \in[0, T), \\ I_{2}, & \text { for } t \in[T, \infty) .\end{cases}
$$

Then $\widetilde{S}_{A}^{s}\left(\mathbf{B}^{2}\right)=S^{0}\left(\mathbf{B}^{2}\right)$, for all $s \geq 0$, but there do not exist $\mathbf{A} \in L\left(\mathbf{C}^{2}\right)$ with $k_{+}(\mathbf{A})<2 m(\mathbf{A})$ and a measurable function $a:[0, \infty) \rightarrow \mathbf{R}$ such that (2.2) holds and $A(t)=a(t) \mathbf{A}$ for a.e. $t \geq 0$.

Proof. Let $s \geq 0$. Since $E+E^{*}=2 I_{2}$, it follows that $\widetilde{S}_{A}^{s}\left(\mathbf{B}^{2}\right)=S^{0}\left(\mathbf{B}^{2}\right)$, by Proposition 4.1. Also, it is elementary to see that there do not exist $\mathbf{A} \in L\left(\mathbf{C}^{2}\right)$ with $k_{+}(\mathbf{A})<2 m(\mathbf{A})$ and a measurable function $a:[0, \infty) \rightarrow \mathbf{R}$ such that $(2.2)$ holds and $A(t)=a(t) \mathbf{A}$ for a.e. $t \geq 0$.

Using Theorem 3.2 and Proposition 4.1, we may prove the following result. This result is also an improvement of Proposition 4.1 (cf. Proposition 2.13 for $\mathbf{A}=I_{n}$ and [13, Theorem 3.12]).

Proposition 4.3. Let $A:[0, \infty) \rightarrow L\left(\mathbf{C}^{n}\right)$ be a measurable mapping such that

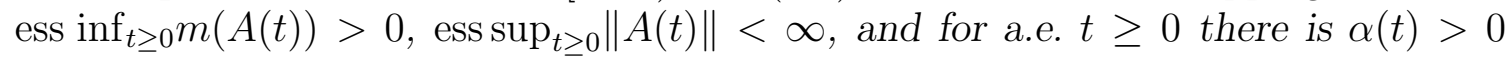
such that $A(t)+A(t)^{*}=2 \alpha(t) I_{n}$. Then $A \in \widetilde{\mathcal{A}}$ and $\widetilde{S}_{A}^{T}\left(\mathbf{B}^{n}\right)=S^{0}\left(\mathbf{B}^{n}\right)$, for all $T \geq 0$.

Proof. Let $\sigma, \beta \in(0, \infty)$ be such that $m(A(t))>\sigma$ and $\|A(t)\|<\beta$, for a.e. $t \geq 0$. In view of the hypothesis, we deduce that there is a measurable function $\alpha:[0, \infty) \rightarrow \mathbf{R}$ such that $\alpha(t)>0$ and $A(t)+A(t)^{*}=2 \alpha(t) I_{n}$, for a.e. $t \geq 0$. We observe that $k(A(t))=m(A(t))=\alpha(t)>\sigma$, for a.e. $t \geq 0$ (cf. [13]).

Fix $T \geq 0$. In view of Lemma 2.3, we have

$$
\left\|V(T, t)^{-1}\right\| e^{-2 \int_{T}^{t} m(A(\tau)) d \tau} \leq e^{-\int_{T}^{t} \alpha(\tau) d \tau} \leq e^{(T-t) \sigma},
$$

for all $t \geq T$, where $V(T, \cdot)$ is the unique solution on $[T, \infty)$ of the initial value problem (2.1) related to $A$. Thus, we deduce that $A \in \widetilde{\mathcal{A}}$. Since $A$ is measurable, there exists a sequence of step functions $\left(A_{k}\right)_{k \in \mathbf{N}}$ defined on $[0, \infty)$ and with values in the set

$$
\left\{E \in L\left(\mathbf{C}^{n}\right) \mid \text { there is } \lambda>\sigma \text { such that } E+E^{*}=2 \lambda I_{n} \text { and }\|E\|<\beta\right\}
$$

such that $A_{k}(t) \rightarrow A(t)$, as $k \rightarrow \infty$, for a.e. $t \in[0, \infty)$. 
Using similar arguments as above, we may prove that for every $k \in \mathbf{N}$, we have

$$
\left\|V_{k}(T, t)^{-1}\right\| e^{-2 \int_{T}^{t} m\left(A_{k}(\tau)\right) d \tau} \leq e^{(T-t) \sigma}
$$

for all $t \geq T$, where $V_{k}(T, \cdot)$ is the unique solution on $[T, \infty)$ of the initial value problem (2.1) related to $A_{k}$. In particular, we deduce that $A_{k} \in \widetilde{\mathcal{A}}$, for all $k \in \mathbf{N}$. Moreover, if we let $\alpha:[T, \infty) \rightarrow \mathbf{R}$ be given by $\alpha(t)=e^{(T-t) \sigma}$, for all $t \geq T$, then $\alpha \in L^{1}([T, \infty), \mathbf{R})$ and thus, by Theorem 3.2, we deduce that

$$
\rho\left(\widetilde{S}_{A_{k}}^{T}\left(\mathbf{B}^{n}\right), \widetilde{S}_{A}^{T}\left(\mathbf{B}^{n}\right)\right) \rightarrow 0, \text { as } k \rightarrow \infty .
$$

Taking into account Proposition 4.1, we conclude that $\widetilde{S}_{A}^{T}\left(\mathbf{B}^{n}\right)=S^{0}\left(\mathbf{B}^{n}\right)$.

In view of Proposition 4.3 and [13, Theorem 3.12], it would be interesting to give an answer to the following question:

Question 4.4. Let $\mathbf{A} \in L\left(\mathbf{C}^{n}\right)$ be such that $k_{+}(\mathbf{A})<2 m(\mathbf{A})$. Also, let $A \in \widetilde{\mathcal{A}}$ be such that $k_{+}(A(t))<2 m(A(t))$ and $S_{A(t)}^{0}\left(\mathbf{B}^{n}\right)=S_{\mathbf{A}}^{0}\left(\mathbf{B}^{n}\right)$, for a.e. $t \geq 0$. Is it true that $\widetilde{S}_{A}^{T}\left(\mathbf{B}^{n}\right)=S_{\mathbf{A}}^{0}\left(\mathbf{B}^{n}\right)$, for all $T \geq 0$ ?

\section{References}

[1] Arosio, L.: Resonances in Loewner equations. - Adv. Math. 227, 2011, 1413-1435.

[2] Arosio, L.: Basins of attraction in Loewner equations. - Ann. Acad. Sci. Fenn. Math. 37, 2012, 563-570.

[3] Arosio, L., F. Bracci, H. Hamada, and G. Kohr: An abstract approach to Loewner chains. - J. Anal. Math. 119, 2013, 89-114.

[4] Arosio, L., F. Bracci, and E. F. Wold: Solving the Loewner PDE in complete hyperbolic starlike domains of $\mathbf{C}^{n}$. - Adv. Math. 242, 2013, 209-216.

[5] Bracci, F.: Shearing process and an example of a bounded support function in $S^{0}\left(\mathbf{B}^{2}\right)$. Comput. Methods Funct. Theory 15, 2015, 151-157.

[6] Bracci, F., M. D. Contreras, and S. Díaz-Madrigal: Evolution families and the Loewner equation II: complex hyperbolic manifolds. - Math. Ann. 344, 2009, 947-962.

[7] Bracci, F., I. Graham, H. Hamada, and G. Kohr: Variation of Loewner chains, extreme and support points in the class $S^{0}$ in higher dimensions. - Constr. Approx. (to appear), doi:10.1007/s00365-015-9302-6.

[8] Dunford, N., and J. Schwartz: Linear operators. Part I: General theory. - Interscience Publishers Inc., New York, 1958.

[9] Duren, P., I. Graham, H. Hamada, and G. Kohr: Solutions for the generalized Loewner differential equation in several complex variables. - Math. Ann. 347, 2010, 411-435.

[10] Elin, M., S. Reich, and D. Shoikhet: Complex dynamical systems and the geometry of domains in Banach spaces. - Dissertationes Math. (Rozprawy Mat.) 427, 2004, 1-62.

[11] Graham, I., H. Hamada, and G. Kohr: Parametric representation of univalent mappings in several complex variables. - Canadian J. Math. 54, 2002, 324-351.

[12] Graham, I., H. Hamada, and G. Kohr: On Subordination chains with normalization given by a time-dependent linear operator. Complex Anal. Oper. Theory 5:3, 2011, 787-797.

[13] Graham, I., H. Hamada, G. Kohr, and M. Kohr: Asymptotically spirallike mappings in several complex variables. - J. Anal. Math. 105, 2008, 267-302.

[14] Graham, I., H. Hamada, G. Kohr, and M. Kohr: Spirallike mappings and univalent subordination chains in $\mathbf{C}^{n}$. - Ann. Sc. Norm. Super. Pisa Cl. Sci. (5) 7, 2008, 717-740.

[15] Graham, I., H. Hamada, G. Kohr, and M. Kohr: Extreme points, support points and the Loewner variation in several complex variables. - Sci. China Math. 55, 2012, 1353-1366. 
[16] Graham, I., H. Hamada, G. Kohr, and M. Kohr: Extremal properties associated with univalent subordonation chains in $\mathbf{C}^{n}$. - Math. Ann. 359, 2014, 61-99.

[17] Graham, I., H. Hamada, G. Kohr, and M. Kohr: Support points and extreme points for mappings with $A$-parametric representation in $\mathbf{C}^{n}$. - J. Geom. Anal. (to appear), doi: $10.1007 /$ s12220-015-9600-z.

[18] Graham, I., and G. Kohr: Geometric function theory in one and higher dimensions. - Marcel Dekker Inc., New York, 2003.

[19] Gurganus, K.: $\Phi$-like holomorphic functions in $\mathbf{C}^{n}$ and Banach spaces. - Trans. Amer. Math. Soc. 205, 1975, 389-406.

[20] Hamada, H.: Polynomially bounded solutions to the Loewner differential equation in several complex variables. - J. Math. Anal. Appl. 381, 2011, 179-186.

[21] Hamada, H.: Approximation properties on spirallike domains of $\mathbf{C}^{n}$. - Adv. Math. 268, 2015, $467-477$.

[22] Hamada, H., M. IAncu, and G. Kohr: Extremal problems for mappings with generalized parametric representation in $\mathbf{C}^{n}$. - Complex Anal. Oper. Theory (to appear), doi: $10.1007 / \mathrm{s} 11785-015-0525-0$.

[23] IANCU, M.: Some applications of variation of Loewner chains in several complex variables. J. Math. Anal. Appl. 421, 2015, 1469-1478.

[24] IAncu, M.: A density result for parametric representations in several complex variables. Comput. Methods Funct. Theory 15, 2015, 247-262.

[25] IANCU, M.: On reachable families of the Loewner differential equation in several complex variables. - Complex Anal. Oper. Theory (to appear), doi:10.1007/s11785-015-0461-z.

[26] Kato, T.: Perturbation theory for linear operators. - Springer, Berlin, 1995.

[27] Pfaltzgraff, J. A.: Subordination chains and univalence of holomorphic mappings in $\mathbf{C}^{n}$. Math. Ann. 210, 1974, 55-68.

[28] Poreda, T.: On the univalent holomorphic maps of the unit polydisc in $\mathbf{C}^{n}$ which have the parametric representation, I-the geometrical properties. - Ann. Univ. Mariae Curie-Skłodowska Sect. A 41, 1987, 105-113.

[29] Poreda, T.: On the univalent holomorphic maps of the unit polydisc in $\mathbf{C}^{n}$ which have the parametric representation, II-the necessary conditions and the sufficient conditions. - Ann. Univ. Mariae Curie-Skłodowska Sect. A 41, 1987, 115-121.

[30] Roth, O.: Control theory in $\mathcal{H}(\mathbf{D})$. - Diss. Bayerischen Univ. Wuerzburg, 1998.

[31] Rотн, O.: Pontryagin's maximum principle for the Loewner equation in higher dimensions. Canad. J. Math. 67, 2015, 942-960.

[32] Schleissinger, S.: On support points on the class $S^{0}\left(\mathbf{B}^{n}\right)$. - Proc. Amer. Math. Soc. 142, 2014, 3881-3887.

[33] Suffridge, T. J.: Starlikeness, convexity and other geometric properties of holomorphic maps in higher dimensions. - In: Lecture Notes in Math. 599, Springer, Berlin, 1977, 146-159.

[34] VodA, M.: Loewner theory in several complex variables and related problems. - Ph.D. Thesis, Univ. Toronto, 2011.

[35] Voda, M.: Solution of a Loewner chain equation in several complex variables. - J. Math. Anal. Appl. 375, 2011, 58-74.

Received 4 August 2015• Accepted 6 December 2015 